\title{
Research on the Application of Network Information Resources in Japanese Translation
}

\author{
Jing Zhang \\ School of Foreign Studies, Xi'an University, 710065
}

\begin{abstract}
Keywords: Network information resources; Japanese translation; Application research
\end{abstract}
\begin{abstract}
On the era of large data and cloud computing, the use of network information resources is a very enthusiastic topic. It is because network information technology and resources gathered in the era of computer using widely and Internet developing rapidly. Based on computer technology, people have put forward new requirements for many disciplines and their own lifestyle. In the traditional language translation, network information technology also plays an important role by its great data advantage. Therefore, it is very important to know how to better use the network information resources in Japanese translation process and based on the important problem of Japanese learning and research to research changes in network information resources and consolidate the practical advancement of the Japanese translation process.
\end{abstract}

\section{Introduction}

Big data era brings both new challenge and the need of changing society for people's life and the form of communication. It can make the networked education and Internet information resources be better used and help people to communicate better and do researches on world development trend. People can resonate when learning foreign language and translation, actively explore and make full use of the massive data generated by computer technology and network information. In the process of communication and progress, people deeply experienced the great impact that network information resources brought to the Japanese translation. Whether it is collecting information or understanding the life of translation objects, massive data and network information resources would have their own unique advantages. These advantages are unimaginable for the people who researched humanistic social science and did word translation job.

\section{The Complexity of Translation Work and the Need for Translation}

For the development of Japanese over the years and the complexity of it as another national language, there must have more binding needs in translation although Japanese has a lot of similarity and proximity to Chinese in many respects. Japanese translation work has its own complexity. This is because Japanese a very unique language developed on the basis of hiragana and katakana. In the process of foreign cultural compatibility, some common language words have formed due to its special cultural background. For Japanese translation work, the professional of translation needs the translator have a variety many capabilities. Language is a tool for communication and language is a necessary way for many enterprises or units to break the barriers of communication in the process of entering the international market. Language is unique to human. When people are expressing especially in the process of Japanese real-time translation, it may be associated with the subjective emotions of themselves. In addition, many translation works have a certain artistic quality. For different language backgrounds and translation requirements, the translation often be more complex and different.

\section{The Conversion of Japanese Original Language and Chinese Cultural Values is Difficult}

Any language would face the differences between source language and translation in people. In other words, language is a manifestation of the transformation between the different cultures of the two countries especially between Japanese and Chinese. As the mother tongue of a nation, Chinese 
is the largest branch of the world's popular language system. It was founded in the era of Emperor Huang in $\mathrm{BC}$ and developed in the late twentieth century $\mathrm{AD}$. It is a kind of language system originated early and matured late. Chinese is a sign of Eastern civilization and result. It is an important information carrier used by humans to precisely name and define things. The system consists of thousands of commonly used words and tens of thousands of idioms which is an indispensable part of civil society. Japanese includes kana and kanji, kana can be divided into hiragana and katakana. The source of Japanese: Japanese is influenced by the radicals of Chinese characters and cursive. Japan was influenced by the culture of Chinese Sui and Tang dynasties, but because Japan took the lead to the international expansion. And in the process of prosperity and expansion, Japan's social system and productivity level gradually developed. Japan's many advanced vocabulary developed at this time. Therefore, although there is a great deal of similarity between Japanese and Chinese, but from the perspective of historical development, it is still different. There is a lot of work to be done in the process of transforming language values and value background systems.

\section{The Accumulation of Information Resources beyond Japanese Translation Skills}

In the process of Japanese translation, in addition to master a lot of vocabulary and accumulate translation skills, the accumulation of information resources beyond Japanese translation is also negligible. This background is particularly true in proprietary Japanese translation or Japanese online translation. For example, for the Japanese special names, place name, Japanese culture, Japanese custom folk culture, some unique wonders in Japan, etc. It can't be finished just rely on translation skills. To avoid the translation errors and ridiculous situation in process of translation, the translator can't be hidebound and just proficient in Japanese words. But should be very familiar with and understand Japanese translation or the cultural background of the paragraph which need to translate. On this basis, using a large number of information resources to achieve a more integrated Japanese translation process.

\section{The Resources that Network Resources can bring to Japanese Translation}

To Facilitate the Search of Japanese Proper Nouns and Academic Terms. Network schedulable resources and practicable information resources is extremely rich. It can provide a convenient inquiry to Japanese proper nouns and academic terms to the maximum extent. As in many areas especially electronic technology, Japan has made great progress, people can clearly find the gap when comparing China with Japan. Japan has walked in front of the world in many areas, it must have its certain creativity. In the process of searching and providing the massive data, people maybe can find the impact for Japanese translation and Japanese traditional areas research from the rapid development of information technology. But these impacts are benign and necessary. You can quickly through the network method to find some of the Japanese special personalization and differences in academic research content. So the translator can be easily to understand the differences between translation content and system with the traditional daily Japanese language translation. Hope the Japanese translation effect can be improved through empirical research methods and innovative spirit. Through bold assumptions and carefully testing the hypothesis, combining with the development of network information, with the convenience of rich network resources to translation and through the network engine query to the name, place name, professional term, the translator can achieve a better translation effect in the practice. The correctness and smooth of the whole sentence can not only rely solely on the separate master on each word, but should be well understood about the practice of background, professional terms and Japanese translation results.

Effective Search for Japanese names, Place Names, Organization Name Translation. In the process of translating names, place names and institutional names in Japan, it is mostly written translation. Or in a negotiation and real-time translation process, the translator may need to understand a certain translation background. Full understand the translation events or translation 
goals. The rapid spread of network information resources and the development of Internet and electronic computer technology to a large extent enriched the advancement of teaching resources. The puzzling content in translation, such as, names, place names proper noun and jargons and so on can be queried through the electronic network search to enter a large database, such as name dictionaries, gazetteers and professional dictionaries, etc. Therefore, in the process of resource extraction, you can enjoy the convenience and advantages of the rapid progress of network and information sharing. For example, the meaning of a vocabulary, people may not understand the lonely language translation process and the meaning of the words in the process of translation. But if you search the vocabulary that you need in the web information engine, you can quickly find the translated content and the answer you need in different translation.

Find the Different Contexts and Original Minds in Japanese Translation. For the translation of Japanese literary works, sometimes behind the translation or art creation of articles often have a very rich story. Creative background, the different created contexts and the creator's mood, emotion and creative intention are likely to have a more important impact on translated documents. The process and results of Japanese translation are based on the creation of Japanese. The times is changing, the same word may be glow with a different life and also have a variety of ways and methods of use. It is because of the progress of the times and the massive language materials accumulated in the large data age make everyone with translation needs can quickly find the original intention of Japanese translator in the process. At least the author's character can be inferred according to the similar of translator. Understanding the original creation motivation is equivalent to solve the problems in Japanese translation process in advance.

\section{Using Network Resources to Fill in the Shortcomings of Traditional Books Information Resources}

Translation is a very complicated job and refers to complex information. In the working process, there are many original translation of Japanese and target translation of language between the language transformation work, and even related to the language development process of religious, life and work practice background. So under the background of big data development, using the network resources to fill the deficiencies of traditional books and information data to broaden the translation channels and provide more convenient translation channels and resources reserves for Japanese translators. Network information resources have become an indispensable assistant for Japanese translators in the translation process. The network information resources can not only improve the translation efficiency and quality of Japanese translators but also make it easy and convenient to get and find translation information. Language is evolving with the development of mankind. In the rapid development of human science and technology, Japanese also in people's production and living environment, there have come out many words that are not commonly used in people's production and living environment. The paper translation dictionaries can't be quickly changed and updated. In the process of professional vocabulary coming out, paper dictionary can't be updated and its storage is limited. And this time the advantages of network are very obvious. Rapid update and reserve energy of the current network information is clearly superior to the traditional information recorded way.

The Fragmentation of Network Information. The fragmentation of network information makes the network information resources update very fast. The update process of much information from the media may be ever-changing or even every second is changing. Many Japanese ordinary people or Japanese professional translation resources of the website update can just provide important information and rich background for the translation. If Japanese translators encounter some realistic problems when translating, you can directly ask the network for help. Web information resources provide a rich information resource library for Japanese translators. And this kind of information resource library is often easy, quick and collecting different aspects of information resource reserves. It can be very clear to make up for the difference between paper dictionaries especially the fragmentation of network information. It can quickly pick up the information without the need for intense translation work in the process of large sections of the 
reference and reading. It provided a more extensive and rich background knowledge platform for the translators. Therefore, nowadays with the rapid development of social science and technology, Japanese translators should have access to the convenience provided by the information age and make use of network information resources for their own translation work.

The Rapidity of Network Information. The rapid development of network information is beyond doubt. Network information is not only more efficient in updating but also very fast in the query. It has brought very much convenience to people's daily life and Japanese translation work. As long as you find the right direction of the query, the well-known query keywords can be very good for supplement the accurate, complete work content of network information resources. What more critical is that this supplementary query work may quickly achieve the purpose within a few seconds. It may only take a short time to complete the previous huge workload even for the more complex part of the entire Japanese translation query work. In the process of network information development, due to the fast update speed, convenient update activities of people, for different network information resources and network information quickly provides the basis for data support, it has provided data support basis for different network information resources and the rapidity of network information. Network information resources can make up for the deficiencies of Japanese translators lack of energy and capacity. It encompasses the words of all walks of life, such as astronomy, geography, history, art, music, cars, daily life and so on. Thus, when a translator touches a translation field that is not familiar or uncommon, it is necessary to have a good understanding of the background of translation in a short time. Under the huge and rich background of the translation resource database the translator can very quickly found the Japanese translation background and methods contained in the network information resources.

Large Base of the Network Resource Update. Massive information and cloud computing has always being the advantages development of network resources. The network information resources of big data age are the rapidly growing of geometric multiple, and constantly absorb the essence of human wisdom to self-improvement process. Today in the use and writing process, people all want their language to be more scientific, reasonable and able to put heads together. In this case, it is necessary for the translator to use the network resources to update the characteristics of large base. In the process of designing with different networks and web pages, the provision of information is often based on massive data.

Different data databases for network information resources technology will have a better help. In the Japanese translation process, it must be built on the basis of rich and extensive data translation considering the source of Japanese language translation content is authoritative and credible, the content of Japanese translation articles and the adaptability of translating customer needs and the promoting function of the whole translation work and purpose for the translation of the practical process. For the same Japanese translation corpus, people may have different understanding and the translation process may vary widely. Therefore, in order to come up with convincing evidence, it is necessary to understand people's practice and find solutions in more databases.

\section{Conclusion}

Although the rapid development of computer science technology and the popularity of computer and networks have given people a certain degree of life challenges, but network information age has brought changes to people's lives, work and learning efficiency. In the context of the Internet age, people can use the network information resources to learn and enhance personal skills. Network information resources also provide unlimited resources and possibilities for language learning. To full use of network information has become a necessary quality and conditions for Japanese translators.

\section{References}

[1] X.F. Gao: Journal of Kaifeng Institute of Education, (2017) No. 2, p 97-98

[2] J. Yang: Research on the Construction of Japanese and Chinese News Corpus Based on Event 
Extraction, (Beijing Jiaotong University, China, 2016)

[3] X.Y. Guo: Technology economy market, (2015) No. 1, p 91

[4] W.L. Zhu: Intelligence, (201444) No. 5, p 192

[5] F.G. Du: Foreign Language Studies in Northeast Asia, (2013) No. 3, p 74-79

[6] W. Shan: Misinterpretation of Translation Practice Report and Its Solution, (Liaoning Normal University, China, 2013)

[7] X.T. He: Technology horizon, (2013), No 6, p 96-126

[8] L. Zhang: Japanese passive expression and translation in "I am a cat", (Jilin University, China, 2012)

[9] Y.W. Zhang: Study of Japanese Translation in "People 's Network" (Ningbo University, China, 2012)

[10] X.P. An: Russian learning, (2011) No 5, p 27-32

[11] J.Y. Zhang: Journal of Southwest University for Nationalities, (2018) No S3, p 110-112

[12]Z. Wang: Cross - cultural Risk Management of International Cooperation Projects, (Kunming University of Science and Technology, China, 2002) 